\title{
La imposibilidad de una metodología científica para el estudio de los textos del periodismo de investigación
}

\author{
José María CAMINos MARCET \\ Universidad del País Vasco UPV-EHU \\ josemaria.caminos@ehu.es \\ Idoia CAMACHO MARQUINA \\ Universidad del País Vasco UPV-EHU \\ idoia.camacho@ehu.es
}

Recibido: 20/04/2010

Aceptado: 25/10/2010

\section{Resumen}

En este artículo se niega la posibilidad de encontrar una metodología científica para el análisis de los textos de investigación publicados en los medios de comunicación. Para alcanzar esta conclusión hemos analizado las técnicas existentes para el estudio de estos textos, así como algunos ejemplos que dejan constancia de las dificultades para alcanzar un procedimiento científico que nos permita afirmar que sin duda nos encontramos ante un artículo de investigación. ¿Cómo podemos afirmar si estamos o no ante un texto investigado cuando los medios tienden a presentar como investigaciones lo que sólo son filtraciones realizadas por una fuente interesada?

Palabras clave: Periodismo de investigación, metodología periodística, fuentes de información, periodismo y filtración, verificación de datos.

\section{The difficulties to implement a scientific methodology in the analysis of investigative journalism articles}

\begin{abstract}
This article denies the possibility of finding a scientific methodology for the analysis of research texts published in the media. In reaching this conclusion we have analyzed existing methodologies for the study of these texts as well as some examples where it is recorded the difficulties of finding a scientific methodology that allows us to clearly state that we have a research text. How can we tell whether or not we have a text under investigation when the media tend to present as what investigations are only conducted by a source leaks interested?
\end{abstract}

Key words: Investigative journalism, journalism methodology, news sources, journalism and filtering, data verification.

\section{Referencia normalizada}

CAMINOS MARCET, José María y CAMACHO MARQUINA, Idoia (2011): "La imposibilidad de una metodología científica para el estudio de los textos del periodismo de investigación". Estudios sobre el mensaje periodístico. Vol. 17, núm. 1, págs.: 43-56. Madrid, Servicio de Publicaciones de la Universidad Complutense

Sumario: 1. Introducción. 2. Estado de la cuestión. 2.1. El problema de la verificación. 2.2. El trabajo con fuentes anónimas. 2.3. Periodismo de filtración-Periodismo de investigación. 2.4. El reconocimiento de que se trata de una filtración. 3. Una propuesta metodológica. 3.1. La oportunidad en la publicación. 4. Un caso significativo: los papeles secretos del Cesid. 4.1. Aplicación de la metodología de estudio a este caso. 4.2. Estudio de la oportunidad en la publicación. 5. Conclusiones. 6. Referencias bibliográficas. 


\section{Introducción}

Uno de los problemas esenciales que tiene un investigador universitario para determinar si se encuentra ante un texto de periodismo de investigación es determinar si la información publicada ha sido ciertamente investigada por el periodista o el medio de comunicación. ¿Existe la certeza absoluta de que el texto es producto de una investigación? ¿Existe la certeza absoluta de que no estamos ante una filtración? Si no existe esa certeza, ¿cómo podemos llevar a cabo un estudio empírico para determinar que los textos analizados son encuadrables en la modalidad del periodismo de investigación?

El recurso permanente del periodismo de investigación a fuentes que no se dejan identificar hace que sea altamente dificultoso llegar a la certeza de que estamos ante un texto de investigación. Este problema se acrecienta si tenemos en cuenta que los periodistas y los medios de comunicación tienden a ocultar las filtraciones periodísticas y en no pocas ocasiones las enmascaran como si fueran informaciones investigadas.

Entre los periodistas practicantes de esta modalidad y los investigadores universitarios que han dedicado sus esfuerzos a analizarla desde una perspectiva científica existe un acuerdo básico: para que exista periodismo de investigación debe haber por parte del periodista investigador una comprobación de los textos publicados a través de diferentes fuentes de información. Eso es lo que hace que un texto se aleje de la simple filtración y se adentre en el campo del periodismo de investigación.

\section{Estado de la cuestión}

\subsection{El problema de la verificación}

Un tema trascendental, por no decir el más importante, a la hora de determinar si nos encontramos ante un texto de investigación es la verificación de los datos publicados.

El periodismo de investigación se sustenta en la constatación de que las informaciones que se publican están perfectamente verificadas por el periodista investigador. Esta es la esencia de su existencia. El periodismo de investigación debe ser incuestionable. Nunca debe caber la más mínima duda de que las informaciones que se publican están suficientemente contrastadas por el periodista investigador.

La clave del periodismo de investigación, lo que lo diferencia de otras prácticas periodísticas que también necesitan de la permanente comprobación de los datos publicados, es que la verificación debe haberla realizado el periodista, y no la fuente de información. Si el periodista -a través de las técnicas propias de esta modalidadverifica los datos publicados, podemos afirmar que estamos ante un texto de investigación. Pero si el periodista publica informaciones ciertas que han sido verificadas por una fuente de información que es la que suministra los datos al periodista para su publicación, estaremos ante una clara filtración.

De esta forma, la verificación de los datos y quién es el autor de esas 
verificaciones se convierte en la piedra angular de esta técnica periodística. Es decir, el trabajo con las fuentes y el trabajo de las fuentes son determinantes para comprobar si estamos ante un texto de investigación.

En este punto tan importante existe consenso en la mayoría de los estudiosos y periodistas que han practicado el periodismo de investigación.

Ferrán Lalueza Bosch (2003: 240), en su tesis doctoral "Periodismo de Investigación televisivo. Marco teórico y metodológico para el análisis de la modalidad”, dirigida por Montserrat QuESADA PÉREZ - experta altamente cualificada en periodismo de investigación y autora de varios artículos y libros sobre el tema-, explica las diferencias entre el periodismo de investigación y el de filtración: "Es posible que un reportaje aporte información inédita esencial, de relevancia permanente y con elementos de denuncia. Sin embargo, si dicha información no ha sido verificada adecuadamente por el periodista, no nos hallamos ante un trabajo de periodismo de investigación, sino que se trata en realidad de una muestra del llamado periodismo de filtración, también conocido en nuestro país -por motivos que no cuesta imaginar- como periodismo de dossier".

También José María CAminos Marcet (1997: 189), autor de un libro sobre periodismo de investigación, aclara estas importantes diferencias: "El verdadero periodismo de investigación es aquél en el que el periodista, a través de su trabajo, su esfuerzo, intuición y sus propias fuentes, descubre algo que el público no conoce y es guardado en secreto. El falso periodismo de investigación es, por el contrario, aquél en el que el profesional no hace sino trabajar sobre unos datos completos que le han sido suministrados por una única fuente de información que exige permanecer en el anonimato. Estas son las fronteras entre el periodismo de investigación y el de filtración”.

Sin embargo, este factor determinante para constatar la existencia de los textos de investigación que es el trabajo con las fuentes de información es de difícil comprobación científica, ya que los investigadores universitarios dependen, en última instancia, de la voluntad del periodista y del medio para reconocer si la información que publican ha sido fundamentalmente investigada por el medio de comunicación o si ha sido esencialmente filtrada por una fuente.

\subsection{El trabajo con fuentes anónimas}

La atribución o no de las fuentes de información en el periodismo de investigación suscita una de las polémicas más candentes de esta modalidad periodística. Hay autores y periodistas que afirman que en el periodismo de investigación no se deben aceptar declaraciones de fuentes que no se dejan identificar. Algunos incluso afirman que nunca aceptan o dan por buenas declaraciones de fuentes que no se dejan atribuir en el texto publicado. Otros autores y periodistas, sin embargo, defienden la posibilidad de publicar las informaciones reveladas por fuentes que exigen mantenerse en el anonimato.

Los defensores de este último punto de vista consideran que un texto es más riguroso, de mayor calidad informativa, si aparece identificada la fuente de información. Desde otra perspectiva, estos mismos autores consideran que con el 
anonimato se secuestra al lector un elemento esencial para valorar el alcance y la autenticidad de las informaciones que el periodista publica. O, lo que es lo mismo, sustraer la identidad de la fuente de información supone privar al lector de un elemento trascendental, ya que el público tiene derecho a saber quién es la fuente que está suministrando los datos. Este punto de vista ya lo compartía Charles SEIB, periodista del Washington Post, cuando afirmaba que "el encubrimiento de las fuentes noticiosas [...] es un juego en contra del público. La prensa y sus miembros habitualmente saben quién está informando [...] Sólo los lectores siguen en la oscuridad. Hace unas décadas, la 'fuente bien informada' y el innombrable 'alto funcionario' eran pájaros raros. Pero hoy esos muchachos reservados pero conocedores están en todas partes” (STRENTZ, 1983: 124).

A pesar del peso real que tienen estas observaciones, es necesario dejar constancia de que en numerosas ocasiones la única posibilidad que tienen los periodistas para llegar a informaciones de interés es a través de fuentes que no se dejan identificar públicamente. Si algunas fuentes consienten en aportar información es porque confían en el periodista y saben que no les va a identificar nunca, pase lo que pase. Desde esta perspectiva podemos decir que es prácticamente imposible que una fuente suministre información confidencial, secreta y de alto riesgo dejándose identificar. Trabajar solamente con fuentes que se dejan identificar sería el fin para el periodismo de investigación.

Luís NúÑEZ LADEvÉZE (1991: 288) considera importante que los datos sobre el tipo de fuente utilizada aparezcan claramente explicados en el primer párrafo, ya que, según afirma, para un lector un texto tiene connotaciones distintas según aparezca o no identificada la fuente de información. Incluso hace una observación que resulta de gran trascendencia para el presente artículo: "Suele ser frecuente en los periodistas ocultar que la iniciativa no proviene de ellos, pues si se da esta circunstancia son conscientes de que el valor informativo del texto queda devaluado".

Estas consideraciones plantean unas cuestiones de interés para el estudio del periodismo de investigación: ¿En manos de quién está la iniciativa? ¿En manos de la fuente? ¿En las del periodista? ¿Se encuentra el público ante una fuente de atribución reservada y no ante una filtración que el periodista oculta y la presenta al público como si se tratara de una fuente anónima?

Las respuestas a estas preguntas nos aportan las claves para diferenciar el periodismo de investigación del periodismo de filtración.

\subsection{Periodismo de filtración-Periodismo de investigación}

Existen unas fronteras perfectamente delimitadas entre lo que entendemos por periodismo de investigación y las filtraciones. Podemos decir con rotundidad que el periodismo de grandes filtraciones no es periodismo de investigación. Eso no excluye, lógicamente, que las filtraciones jueguen un importante papel en el periodismo de investigación.

Habitualmente todos los periodistas trabajan con filtraciones y más los que practican el periodismo de investigación. En esta modalidad periodística las 
filtraciones siempre están ahí pero, en su actividad, el periodista siempre debe analizarlas y comprobarlas.

Ahora bien, los periodistas que han practicado esta modalidad y los estudiosos del tema coinciden en un aspecto esencial para decidir si estamos ante un trabajo de investigación y es que para considerar un texto como tal, la investigación y verificación de los datos ha tenido que estar en manos del periodista, y no exclusivamente en manos de la fuente de información.

Si la verificación de los datos ha estado en manos exclusivamente de la fuente nos encontramos ante una filtración. Si ésta es total, es decir si la fuente suministra al periodista unos datos concluidos y verificados en su totalidad solamente por ella, nos encontramos ante una gran filtración, pero no ante un texto de investigación.

El trabajo con filtraciones es importante en el periodismo de investigación, pero sólo podremos considerar un trabajo como de investigación cuando la filtración ha servido exclusivamente para poner al periodista sobre el tema, para ofrecerle pistas, para orientarle en su trabajo, pero la verificación de los datos ha sido realizada por el periodista.

Es más, numerosos periodistas investigadores coinciden en afirmar que las grandes filtraciones para que puedan ser publicadas deben ser previamente comprobadas por el periodista. No es suficiente con que las revelaciones que se publican hayan sido verificadas por la fuente. Si el periodista no lo hace así, asume la responsabilidad de poder estar publicando textos con una finalidad desinformadora. Sin embargo, a pesar de estas afirmaciones, coinciden también en afirmar que demasiado frecuentemente nos encontramos ante textos que han sido totalmente filtrados y que no han sido sometidos a una estricta verificación.

Queremos dejar claro que no existen problemas éticos o deontológicos para publicar informaciones totalmente filtradas que son veraces, porque están suficientemente comprobadas. Pero estas informaciones no son periodismo de investigación porque la verificación ha estado en manos de la fuente y no del periodista investigador. Se trata de periodismo de filtración.

Para que exista periodismo de investigación la verificación ha tenido que ser fruto del trabajo profesional del periodista que, merced al uso de unas técnicas específicas, ha verificado y comprobado los datos. Es decir, en el caso de que haya existido filtración, ésta ha tenido que ser parcial y colocar al periodista sobre la pista de unas informaciones que posteriormente se han verificado gracias a su trabajo investigador.

Este punto de vista lo encontramos en CAminos (1997: 189) cuando afirma que "el verdadero periodismo de investigación es aquel en el que el periodista, a través de su trabajo, su esfuerzo, su intuición y sus propias fuentes, descubre algo que el público no conoce y es guardado en secreto. El falso periodismo de investigación es, por el contrario, aquél en el que el profesional no hace sino trabajar sobre unos datos completos que le han sido suministrados por una única fuente de información que exige permanecer en el anonimato".

Estas diferencias entre un tipo y otro de periodismo son muy importantes. El 
periodismo de dossier no es periodismo de investigación, el periodismo de cheque pagado no es periodismo de investigación, el periodismo de filtración no es periodismo de investigación. La frecuente equiparación de estas diferentes modalidades plantea un obstáculo casi insalvable para los investigadores universitarios: ¿cómo pueden reconocer que el texto publicado es una investigación y no una filtración que el medio de comunicación ha presentado como un texto investigado?

\subsection{El reconocimiento de que se trata de una filtración}

Está claro que algunos medios de comunicación han publicado y publican informaciones filtradas y las presentan a los lectores como si se tratara de textos de investigación, como si fueran textos que han sido elaborados tras un minucioso trabajo investigador. Es más, es conocido y notorio que algunos medios que han practicado el periodismo de investigación han publicado alguna vez filtraciones que han presentado al público como si se tratara de trabajosas investigaciones llevadas a cabo por sus periodistas.

La publicación de estas informaciones filtradas que se presentan al público como textos investigados contraviene las normas éticas y deontológicas de la práctica profesional periodística, porque se miente al público en un aspecto esencial: el reconocimiento de las fuentes de procedencia de la información.

Para el público receptor de los medios de comunicación, es muy importante conocer si las revelaciones publicadas provienen de un dossier filtrado o de una investigación. Tal y como explica HÉCTOR BORRAT (1989: 72), a través de estos enmascaramientos "los medios de comunicación han logrado acostumbrar a sus audiencias al consumo indiscriminado y casi cotidiano de informaciones filtradas que no se presentan como tales sino como simples componentes del flujo informativo normal”. Desde esta perspectiva es necesario recordar que las grandes filtraciones tienen casi siempre intencionalidades ocultas y es muy importante que el público conozca este dato para valorar en su justa medida el alcance de esa información.

$\mathrm{Y}$ es aquí cuando los investigadores universitarios nos encontramos ante un obstáculo prácticamente insalvable para determinar si efectivamente estamos ante un texto de investigación, lo que hace que sea prácticamente imposible estudiar con precisión científica la autenticidad de los textos relacionados con el periodismo de investigación.

\section{Una propuesta metodológica}

Consciente de las dificultades para llevar a cabo un estudio científico de los textos de investigación, Ferrán LaLUEZA Bosch, en la tesis anteriormente citada, aporta un marco teórico para el análisis de la modalidad y expone una metodología para el estudio científico de los textos que pueden formar parte del periodismo de investigación.

El gran valor de esta tesis doctoral es que la propuesta metodológica de Lalueza es la primera que se ofrece en la Periodística española para determinar si nos 
encontramos ante un texto de investigación y así intentar abrir las puertas a la investigación científica de los textos publicados por la prensa en materia de periodismo de investigación. Hasta el momento ningún otro autor ha planteado una propuesta alternativa o que amplíe y perfeccione esta metodología, por lo que para estudiar los textos de investigación tenemos que referirnos necesariamente a ella.

Ferrán LaLuEza (2003: 226) propone el estudio de unos factores que "deben caracterizar a una información para que el trabajo periodístico que la aporta pueda considerarse investigativo" y los concreta en estos cuatro: 1) que la información sea verificada; 2) que sea inédita esencial; 3) que sea de relevancia permanente y 4) que sea denunciadora.

Cuando LALUEZA propone la confluencia de estas cuatro características está dejando claro que la presencia de una o de algunas de ellas no son suficientes para definir un texto de investigación, sino que es necesaria la confluencia de todas y cada una de las ellas.

Vamos a referirnos inicialmente a los tres últimos factores y finalmente reflexionaremos sobre el factor que el autor de la tesis denomina información verificada:

Información inédita esencial: Lo inédito por sí solo no sirve para tipificar un texto como de investigación. Lo inédito lo podemos vincular a lo novedoso y ésta es una característica de cualquier hecho noticioso, no exclusivamente de los de investigación. Por eso Ferrán LALUEza (2003: 231) propone vincular al concepto de inédito el adjetivo esencial. Según explica este autor, el carácter esencial "implica cierta iniciativa por parte del periodista” ya que una información rigurosamente inédita "supone un meritorio rasgo diferenciador más allá del valor periodístico que tradicionalmente se ha dado a la primicia”.

Información de relevancia permanente: Hablar de relevancia permanente supone para este autor excluir de la modalidad "los trabajos que se centran en lo frívolo, lo morboso, lo anecdótico y lo insustacial”, es decir, en palabras de NúÑEz LADEVÉZE, prescindir de las temáticas de interés del público para centrarse en los temas de interés público. Esta característica conecta también con la idea de que un reportaje investigativo tiende a crear repercusiones. Afirma LALUEZA (2003: 235) que "cuanto mayor sea la relevancia de la información aportada mayores serán las posibilidades de que su difusión produzca efectos observables”.

Información denunciadora: Aunque no todo el periodismo de denuncia es investigativo, el componente denunciativo siempre ha estado asociado al periodismo de investigación. No hay que olvidar, según este autor, "que a menudo puede denunciarse un hecho o una situación que, aun siendo alegal o incluso perfectamente legal, debe considerarse ilícito" (2003: 236). El periodismo de investigación se centra en la denuncia de actividades que pueden considerarse ilícitas.

Información verificada: Para Ferrán LALUEZA (2003: 229), “un trabajo de investigación periodística sólo debe informar sobre aquellos hechos cuya veracidad haya sido rigurosamente verificada por el reportero". "Desde el momento en que el reportero ha recolectado -a través de un proceso investigativo metodológicamente 
solvente- las certezas suficientes para que su información no pueda ser rebatida fehacientemente podemos considerar que la información ha sido debidamente verificada". "La necesidad de verificar todos los hechos es ampliamente aceptada por los estudiosos y practicantes de la modalidad y constituye -ya de entrada- uno de los pilares básicos de su metodología especializada, de esa metodología que vertebra nuestra concepción del periodismo investigador” (2003: 230).

Nos encontramos aquí ante un elemento esencial para calificar un texto como de investigación, pero también ante un escollo prácticamente insalvable, ya que los medios, como hemos explicado, ocultan en numerosas ocasiones si el texto publicado es una filtración y tienden a presentar como investigado por el periodista y el medio un texto que simplemente ha sido filtrado y cuya verificación ha estado en manos de la fuente, pero no en las del periodista ni en las del medio de comunicación.

\subsection{La oportunidad en la publicación}

Como comprobaremos posteriormente, existen serias dificultades para que una vez aplicada esta metodología propuesta por LALUEZA tengamos argumentos suficientes para determinar sin posibilidad de error si nos encontramos o no ante un texto de investigación.

El aspecto más complicado lo encontramos a la hora de demostrar, más allá de lo que afirma el medio de comunicación, si estamos ante una investigación periodística o ante una filtración. Para poder aclarar este punto con mayor rigor consideramos oportuno incluir una nueva variable a esta metodología. Se trata de analizar el momento en el que se hacen públicas las informaciones; es decir, la oportunidad de la publicación. Para ello proponemos vincular la información publicada al contexto global en el que se produce la publicación. Esta vinculación contextual, el estudio de la oportunidad en la publicación, no arrojará claves importantes para determinar si nos encontramos ante una investigación, pero sí nos dará pistas más contundentes para sospechar que nos podemos encontrar ante una filtración.

Sobre el momento de la publicación de los textos de investigación podemos decir que, a diferencia de otros tipos de textos, incluso de otras informaciones exclusivas, no es necesario esperar a un momento oportuno para su publicación. Los textos de investigación, en tanto en que sacan a la luz hechos ocultos, crean su propia actualidad, ya que a pesar de que no están vinculados al acontecer reciente -tratan siempre sobre temas pasados-, sí lo están al descubrimiento reciente, por lo tanto tienen una permanente actualidad, lo que hace que no sea necesario esperar a un momento oportuno para su publicación. Si se hace así, puede ser porque el medio de comunicación busca unos efectos estratégicos que van más allá de la simple publicación. Puede ser, por ejemplo, que quiera presionar a alguien con una intención oculta.

Por eso consideramos importante analizar la vinculación contextual. Las filtraciones siempre son interesadas y se efectúan en momentos estratégicos importantes para la fuente filtradora. De acuerdo con NúÑEZ LADEVÉZE (1991: 287) podemos definir la filtración como "el suministro interesado o por encargo de sus 
superiores de información por una fuente que mantiene el anonimato no porque el contenido de la información le perjudique sino porque infringe la obligación de guardar sigilo".

También Petra María SeCANella (1986: 98) se refiere a la filtración como de una técnica altamente intencional. Para esta autora, "filtrar es una política muy bien pensada por los funcionarios que quieren influir sobre una decisión política, promover una línea de actuación, persuadir al Legislativo [...]”.

Una característica esencial de las filtraciones es que siempre aparecen en momentos de crisis y conflictos. Los filtradores comienzan su trabajo cuando las posturas en conflicto llegan a su máximo grado de enfrentamiento y con un claro objetivo: influir en la opinión pública mediante informaciones que, por un motivo u otro, no podrían darse a conocer oficialmente.

El periodista de El País Carlos YÁRNoz vincula esta política informativa fundamentada en el uso de filtraciones con la intención de influir en decisiones políticas importantes, tal y como recoge CAminos (1997: 190). "Nadie piensa a estas alturas que fue producto de la casualidad el hecho de que las conversaciones o negociaciones que Mario Conde sostuvo con el Gobierno entre el 24 de febrero y el 1 de septiembre de 1995 coincidieran, en los momentos claves de las mismas, con la difusión de escandalosas noticias sobre los GAL y el Cesid a través del diario El Mundo. Tampoco nadie se refugia en la casualidad cuando se comprueba que esas noticias correspondían precisamente con los documentos sustraídos hace años del Cesid por el ex agente Juan Alberto Perote, personaje muy ligado a Mario Conde”.

La vinculación de la noticia publicada con el contexto en el que se produce su publicación no resuelve el tema de si estamos ante una información investigada o ante una información filtrada, pero sí arroja pistas claras para que el investigador universitario pueda sospechar que se encuentra ante una filtración.

\section{Un caso significativo: Los papeles secretos del Cesid}

El 16 de diciembre de 1996 el diario El Mundo inició la publicación de unos documentos secretos que el Gobierno se había negado a entregar a la Justicia y que podrían implicar a distintos responsables de la Seguridad del Estado durante la etapa de Felipe González en la guerra sucia contra ETA. Finalmente, tras una primera negativa, el gabinete presidido por Aznar aprobó desclasificar los denominados “papeles del Cesid” en abril de 1997.

Dichos papeles secretos llegaron a marcar la agenda política entre los años 1995 y 1997, ya que la Audiencia Nacional solicitó en varias ocasiones su desclasificación por parte del Gobierno, petición que también realizaría el Tribunal Supremo.

El mismo día de su publicación El Mundo publicó un editorial titulado "Al fin, todos los papeles del Cesid", en el que afirmaba: "Un muy intenso trabajo de investigación periodística, desarrollado durante más de un año, nos ha permitido localizar los documentos que no se conocían y ratificar la veracidad de los que ya habíamos publicado". 
El 16 de diciembre de 1996 este diario abrió su portada con una información a cuatro columnas cuyo antetítulo recogía la frase siguiente: "El Mundo inicia hoy la publicación de todos los documentos secretos que el Gobierno se niega a entregar a la Justicia”. En el titular de esta noticia se aseguraba que "El Cesid seleccionó como objetivo al primer etarra asesinado por los GAL”.

Figura $n^{\circ} 1$

\section{EL来MUNDO}

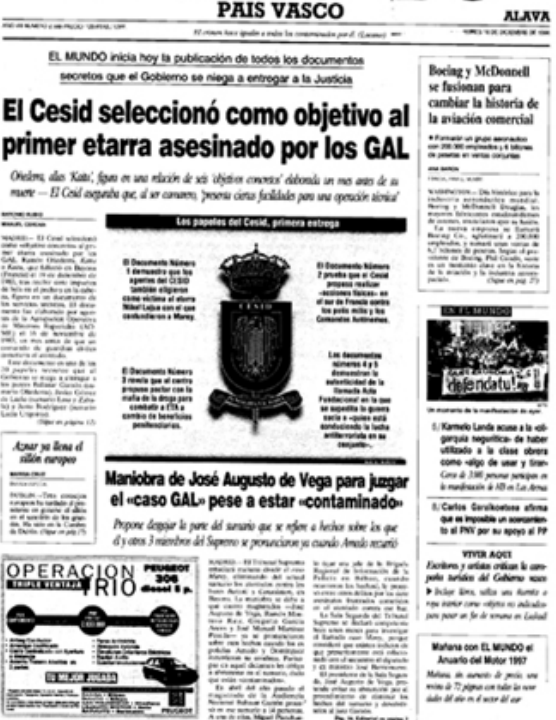

El Mundo ofreció el 16 de diciembre de 2010 la primera entrega de los papeles del CESID.
Figura $n^{\circ} 2$

\section{EL PAIS}

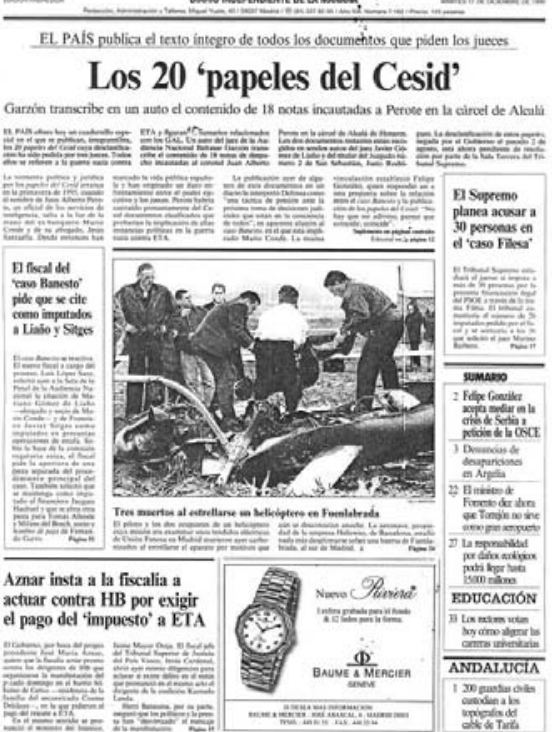

El País publicó el 17 de diciembre de 2010 el documento en su totalidad

Al día siguiente, esto es, el 17 de diciembre de aquel año, el diario El País abrió su portada con un texto a cinco columnas con el antetítulo siguiente: "El País publica el texto íntegro de todos los documentos que piden los jueces”. En el titular de esta noticia se podía leer la frase siguiente: "Los 20 papeles del Cesid".

Al parecer, según afirmaba el diario El País aquel día, los papeles secretos del Cesid habrían sido sustraídos presuntamente por Juan Alberto Perote, un oficial de los servicios secretos del espionaje español, que mantenía vinculaciones con el expresidente de Banesto Mario Conde. El banquero se enfrentaba en aquellas fechas a un juicio por apropiación indebida de fondos de dicha entidad financiera (caso Argentia Trust), por el que resultaría condenado a cuatro años de cárcel.

De acuerdo con El País, "la publicación ayer de algunos de esos documentos en un diario la interpretó Defensa como una táctica de presión ante la próxima toma de decisiones judiciales que están en la conciencia de todos” (El País, 17 de diciembre de 1996). También el ex-presidente del Gobierno, Felipe González, vinculó la publicación de los papeles del Cesid con el juicio contra Mario Conde con la frase siguiente: "No hay que ser adivino; parece que coincidir, coincide" (El País, 17 de diciembre de 1996). 
Ese mismo día, El País dedicó uno de sus editoriales a este tema en el que afirma que "los papeles del Cesid que hoy publica El País [...] son fruto de la requisa que practicó Garzón en la celda que el coronel Perote ocupa en la prisión militar de Alcalá de Henares”. [...] Al publicar estos papeles, íntegramente y de una vez, EL PAÍS intenta, ante todo, acabar con cualquier pretensión de administrar a conveniencia la agenda política mediante la difusión dosificada de informaciones o documentos comprometedores. El diario El Mundo publicó ayer cinco de esos papeles y anunció nuevas remesas para días sucesivos. Pues bien, aquí están todos. El muy intenso trabajo de investigación periodística a que aludía ayer ese diario consiste en ser recipiendario de una filtración. Que la fecha de difusión se elija en función de otro sumario, el del caso Banesto, que ayer vivió una sesión singular, es una sospecha no probada, pero en absoluto carente de fundamento".

Es decir, el 16 de diciembre El Mundo publicó, gracias a "un muy intenso trabajo de investigación periodística, desarrollado durante más de un año", unos papeles a los que también tuvo acceso el diario El País, que los hizo públicos un día más tarde. Por tanto, el diario El Mundo presentó como un trabajo de investigación algo que, según su rival El País, no era sino una mera filtración.

Sin embargo, a la luz de los argumentos que hemos planteado en el presente artículo, podemos decir rotundamente que El Mundo no habría contravenido ninguna norma ética o deontológica por publicar unas informaciones supuestamente filtradas si las mismas eran veraces. Sí lo habría hecho, en cambio, al presentar dichas informaciones como producto de una ardua investigación, cuando cabe la sospecha de que se tratara de una filtración.

Esta circunstancia es altamente significativa para los investigadores universitarios que quieran estudiar los textos de investigación y plantea unas cuestiones muy importantes: ¿Cómo sabemos que un texto publicado por un medio responde a las características que hemos atribuido al periodismo de investigación? ¿Cómo sabemos que estamos ante una investigación y no ante una filtración?

No existe un método que nos permita determinar esta circunstancia sin temor a equivocarnos, sólo contamos con la palabra del periodista y del medio de comunicación. Ningún estudio sobre el texto publicado o las fuentes utilizadas pueden resolver estas dudas más allá de lo que el medio quiera afirmar. Vistas así las cosas, no nos queda más remedio que preguntarnos ¿cómo podemos utilizar un procedimiento científico, sin posibilidad de error cuando sabemos, como en el ejemplo presente, que algunos medios tienden a ocultar que publican filtraciones y las presentan como textos de investigación para así incrementar su credibilidad y prestigio?

\subsection{Aplicación de la metodología de estudio a este caso}

Para comprobar la validez de la metodología propuesta por Ferrán LALUEZA para el estudio de los textos de investigación, hemos aplicado sus aportaciones a la información publicada en su día por el diario El Mundo sobre los papeles secretos del CESID: 
¿La información publicada es inédita esencial?: Sí, sin lugar a dudas se aportaba información que en esos momentos era inédita. Es más, varios jueces de la Audiencia Nacional habían solicitado al Gobierno que aportara los citados documentos, lo que no sucedería hasta abril de 1997. Sacar a la luz dichos papeles suponía aportar una información que era inédita y, además, esencial para el curso de las investigaciones judiciales.

¿Se trata de un texto de relevancia permanente?: El texto publicado por El Mundo era de gran relevancia. Hay que tener en cuenta que en el momento de la publicación de los mismos se habían iniciado ya algunos sumarios contra importantes responsables del Ministerio del Interior, que acabarían siendo condenados a prisión. La publicación de los papeles secretos del Cesid hizo que durante meses se produjera una cascada de informaciones relacionadas con el tema.

¿La información implica una denuncia?: Claramente sí. El texto es una denuncia muy importante de actividades ilícitas llevadas a cabo por los Servicios de Seguridad del Estado, durante la etapa de Felipe González, para combatir el terrorismo de ETA y deja clara la directa implicación de los mismos en la creación del GAL y en las acciones posteriores protagonizadas por dicho grupo.

¿La información está suficientemente verificada?: La información estaba perfectamente verificada. El Mundo publicó informes secretos de los servicios de inteligencia del Estado. En sus artículos aportó fotocopias de los documentos para dotar de credibilidad a los textos.

De acuerdo con la metodología que aporta LALUEZA para la contemplación de los textos de investigación, hay que concluir que estábamos sin lugar a dudas ante el fruto de una investigación. Si el diario El País no hubiera publicado un día más tarde los mismos documentos no podríamos sospechar de que podíamos encontrarnos ante una filtración.

Nos situamos así ante el problema fundamental para determinar si un texto es o no de investigación. De acuerdo con sus características textuales es fácil determinar si se trata de un texto inédito esencial, de relevancia permanente y de denuncia. También si está o no suficientemente verificado. Lo que no podemos concretar es si la verificación ha sido realizada por el medio de comunicación o por la fuente, aspecto éste esencial para determinar si estamos ante un texto de investigación. Si el medio de comunicación oculta la filtración interesada y presenta el texto como una investigación, los investigadores universitarios carecemos de un dato esencial, que imposibilita el estudio científico de los textos de investigación.

\subsection{Estudio de la oportunidad en la publicación}

Si vinculamos a su contexto la información publicada por El Mundo sobre los papeles del CESID, aparece una variable nueva que nos ayuda a sospechar que nos podemos encontrar ante un texto filtrado. ¿Existe oportunidad en la publicación de esa información? Claramente sí.

Los papeles del Cesid aparecen publicados en un momento en el que Mario Conde tiene varios sumarios abiertos en relación con apropiación indebida de 
fondos de Banesto, banco del que fue presidente desde 1987 hasta diciembre de 1993, en que la entidad fue intervenida por el Banco de España. En marzo de 1997 la Audiencia Nacional condenó a Conde a seis años de prisión por el caso Argentia Trust (el Tribunal Supremo rebajaría posteriormente la pena a cuatro años). Según publicó la prensa de la época, Mario Conde mantuvo contactos con el coronel Juan Alberto Perote, ex dirigente del Cesid, que tuvo en sus manos los papeles que posteriormente hizo públicos el diario El Mundo.

Si analizamos la vinculación contextual, el momento en el que aparecen publicadas esas informaciones, podemos sospechar que estamos ante una filtración y añadir así una duda razonables a la hora de determinar si nos encontramos ante un texto investigado por el periodista.

En cualquier caso, insistimos una vez más que este estudio nos enfrenta ante la sospecha, pero no sirve para determinar de forma contundente si nos encontramos o no ante una filtración.

El periodismo de investigación no es periodismo especializado. Es sin más una técnica para el trabajo periodístico. Un trabajo que se dota de unas técnicas específicas para llegar a informaciones ocultas y que para su estudio científico requiere la honestidad de los medios de comunicación para informar al público si los textos publicados son filtraciones o investigaciones, independientemente de que se identifique o no a las fuentes de información.

Como explica Javier Chicote (2006: 72), “el periodismo de investigación está siendo sistemáticamente atacado y desvirtuado por las múltiples apropiaciones indebidas del término que abundan en los medios de comunicación. Se trata de filtraciones e informaciones poco rigurosas, superficiales y sensacionalistas que se venden al público como si de investigaciones se tratara”.

Con estas aclaraciones metodológicas no pretendemos echar por tierra la propuesta de Ferrán LALUEZA, cualquier otra metodología debería ofrecer la completa seguridad de que si un medio afirma que está publicando informaciones profundamente investigadas realmente es así, y como hemos podido comprobar con este ejemplo concreto nunca tenemos la certeza absoluta de que nos encontramos ante un texto que realmente ha sido investigado por el medio de comunicación.

Por eso no tenemos más remedio que afirmar que es imposible aplicar una metodología científica absolutamente independiente porque dependemos de la voluntad del medio de comunicación y siempre nos vamos a encontrar ante un muro insalvable: ¿el texto estudiado es realmente una investigación o se trata de una filtración que el periodista presenta como investigación? Cualquier otra metodología se enfrentaría ante este mismo dilema.

El trabajo con fuentes anónimas forma parte de la esencia del periodismo de investigación. Es más, sin el compromiso del periodista para no revelar la identidad de la fuente no existiría el periodismo de investigación. Pero apelar a fuentes anónimas puede ser una estratagema del medio para ocultar filtraciones. Nunca tendremos la seguridad de que no es así, por lo que el trabajo científico de los textos de investigación se presenta como una tarea inalcanzable. 


\section{Conclusiones}

1.- El periodismo de investigación no forma parte del periodismo especializado, no es más que una técnica de trabajo periodístico cuya finalidad es sacar a la luz temas ocultos.

2.- Para que exista periodismo de investigación es necesario que la verificación de los datos la haya realizado el periodista, y no la fuente de información. Si es el periodista el que verifica los datos nos encontramos ante una investigación. Si la fuente es la que los ha verificado nos encontramos ante una filtración.

3.-Las metodologías propuestas para la constatación de que estamos ante un texto de investigación chocan siempre ante un muro insalvable: ¿Cómo tenemos la seguridad de que no se trata de una filtración si los medios tienden a presentar las filtraciones como producto de una investigación?

4.- Ningún análisis textual ni de las fuentes utilizadas ofrecen la seguridad de que nos encontramos ante un texto de investigación. De ahí que podamos concluir que no es posible establecer una metodología para el estudio de los textos de investigación que garantice que no nos encontramos ante una filtración que el medio de comunicación presenta como una investigación.

\section{Referencias bibliográficas}

BORRAT, Héctor (1989): El periódico, actor político. Barcelona, Gustavo Gili.

CHICOTE, Javier (2006): "Los enemigos del periodismo de investigación”, en Estudios sobre el Mensaje Periodístico, $\mathrm{n}^{\circ} 12$. Madrid, Servicio de Publicaciones de la Editorial Complutense, pp. 71-90.

CAMINOS MARCET, José María (1997): Periodismo de Investigación: Teoría y Práctica. Madrid, Síntesis.

LALUEZA, Ferrán (2003): Periodismo de Investigación televisivo. Marco teórico y metodológico para el análisis de la modalidad. Tesis Doctoral leída en la Universidad Pompeu Fabra de Barcelona.

NÚÑEZ LADEVÉZE, Luis (1991): Manual para periodismo. Barcelona, Ariel.

QUESADA, Montserrat (1987): La investigación periodística. El caso español. Barcelona, Ariel Comunicación.

SECANELLA, Petra María (1986): Periodismo de investigación. Madrid, Tecnos.

STRENTZ, Herbert (1983): Periodistas y fuentes informativas. Buenos Aires, Marymar. 